\title{
XMM-Newton imaging of V1818 Orionis: a young stellar group on the eastern edge of the $\kappa$ Ori ring ${ }^{\star}$
}

\author{
I. Pillitteri ${ }^{1}$, S. J. Wolk ${ }^{2}$, and S. T. Megeath ${ }^{3}$ \\ 1 INAF-Osservatorio Astronomico di Palermo, Piazza del Parlamento 1, 90134 Palermo, Italy \\ e-mail: pilli@astropa.inaf.it \\ 2 Harvard-Smithsonian Center for Astrophysics, 60 Garden St, Cambridge (MA), 02138, USA \\ 3 Ritter Astrophysical Research Center, Dept. of Physics and Astronomy, University of Toledo, Toledo, OH, USA
}

Received 2 June 2017 / Accepted 13 November 2017

\begin{abstract}
We present the results of a $40 \mathrm{ks} X M M$-Newton observation centered on the variable star V1818 Ori. Using a combination of the XMM-Newton and AllWISE catalog data, we identify a group of about 31 young stellar objects (YSOs) around V1818 Ori. This group is coincident with the eastern edge of the dust ring surrounding $K$ Ori. Previously, we concluded that the YSOs on the western side of ring were formed in an episode of star formation that started 3-5 Myr ago and are at a distance similar to that of $k$ Ori $(250-280 \mathrm{pc})$ and in the foreground to the Orion A cloud. Here we use the XMM-Newton observation to calculate X-ray fluxes and luminosities of the young stars around V1818 Ori. We find that their X-ray luminosity function (XLF), calculated for a distance of $\sim 270 \mathrm{pc}$, matches the XLF of the YSOs west of $\kappa$ Ori. We rule out that this group of young stars is associated with Mon R2 as assumed in the literature; they are instead part of the same stellar population of the $\kappa$ Ori ring.
\end{abstract}

Key words. stars: activity - stars: formation - stars: individual: V1818 Ori, $\kappa$ Ori

\section{Introduction}

Understanding the spatial structure and distribution of young stellar objects (YSOs) in the Orion molecular cloud complex is crucial for tracing its star formation history. The Orion OB association is characterized by several subgroups of stars of different masses, ages, and distances; its southern portion, Orion A, has distances of between $\sim 388$ pc and 428 pc (Kounkel et al. 2017; Bally 2008; Menten et al. 2007). The Orion region thus offers a wide spectrum of test cases for various mechanisms of star formation, such as self-collapse vs. triggered star formation, the interplay of external agents as opposed to in situ evolution, or the formation of low-mass groups in isolation vs. mass-segregated formation in denser and more massive clusters.

In this context, we investigated the X-ray emission of YSOs west of $\kappa$ Ori and south of Orion A/L1641 Pillitteri et al. (2016). These young stars lie within a ring of gas and dust that apparently surrounds the B0 (V) star $\kappa$ Ori within a radius of $2 \mathrm{deg}$ (Fig. 1). The ring appears as the rim of a bubble created by the sweeping action of the stellar winds emanating from $\kappa$ Ori. This structure overlaps the L1641 region of the Orion A cloud. However, the quoted distance to $\kappa$ Ori is about 200-240 \pm 40 pc (Megier et al. 2009; van Leeuwen 2007), much closer than Orion A (around $400 \mathrm{pc}$ ). We therefore investigated the relationship between the YSOs in the ring and Orion A. By means of X-ray observations of the YSOs in the ring of dust and by comparing their $\mathrm{X}$-ray luminosity function (XLF) to that of L1641 and ONC stars, we determined that the distance to the YSOs near $\kappa$ Ori is about $250-280 \mathrm{pc}$, similar to the $\kappa$ Ori distance, and they are

\footnotetext{
* Based on observations obtained with XMM-Newton, an ESA science mission with instruments and contributions directly funded by ESA Member States and NASA.
}

therefore unrelated to Orion A. They are instead a distinct population of YSOs born within the ring of gas and dust that is centered on $\kappa$ Ori, as is visible in extinction maps and mid-IR and submillimeter images.

V1818 Ori is a Herbig Be star surrounded by about two dozen YSOs, and it apparently lies on the eastern edge of the same ring of dust that is centered on $\kappa$ Ori (Fig. 1). V1818 Ori has highly irregular variability and bursts (Chiang et al. 2015), and it is a binary system with components separated by $3.5^{\prime \prime}$. The spectra show strong veiling that might be due to active accretion, and the stellar variability might also affect the determination of its spectral type, which is B7 as reported by Chiang et al. (2015), but is F0 as classified by Connelley \& Greene (2010). Based on the similarity of the velocities measured from stellar absorption lines with that of the gas toward the Mon R2 association, Chiang et al. (2015) assumed that these YSOs are part of the Mon R2 star-forming complex at a distance of $900 \mathrm{pc}$. However, given its coincidence with the $\kappa$ Ori ring, the question of the distance to V1818 Ori remains unanswered.

In the present paper we analyze an XMM-Newton observation centered on V1818 Ori to resolve the question of the distance to V1818 Ori and its nearby YSOs, to search for evidence of young stars to the east of $\kappa$ Ori and to establish the extent and spatial structuring of the ongoing star formation in the ring.

The paper is structured as following: in Sect. 2 we present the observation and the data analysis, in Sect. 3 we present and discuss our results, and we conclude in Sect. 4.

\section{Observation and data analysis}

We observed the region toward V1818 Ori with XMM-Newton on April 6, 2017, for a nominal duration of $40 \mathrm{ks}$ (PI: Ignazio 


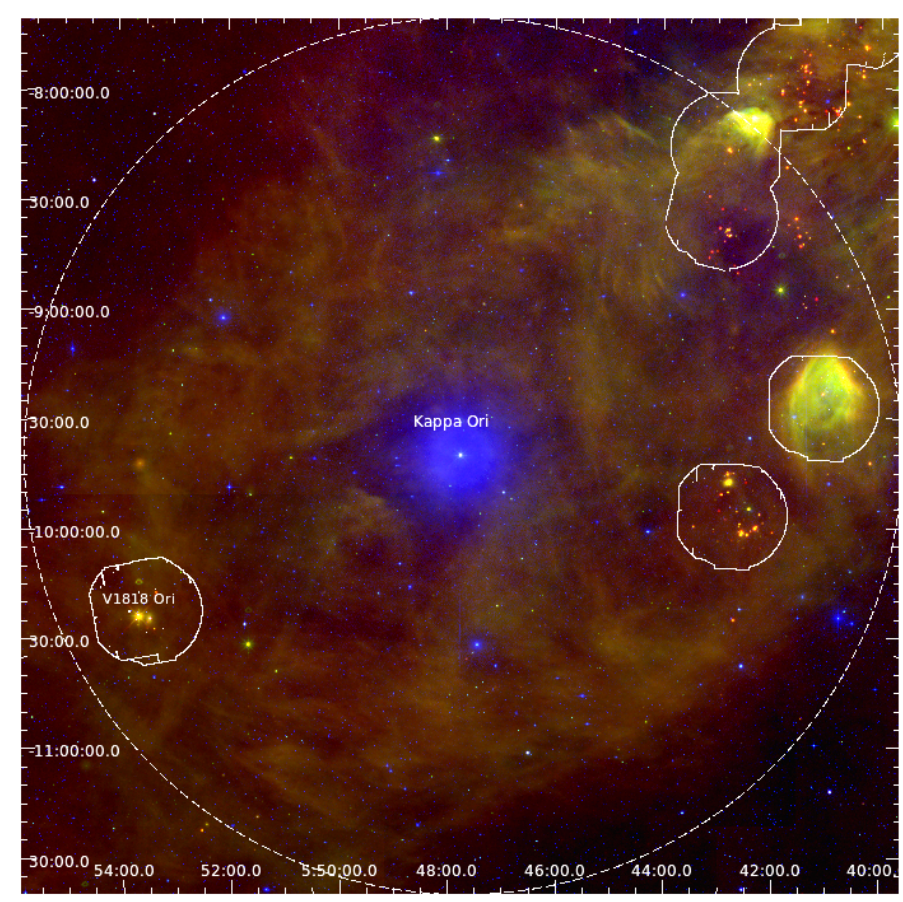

Fig. 1. Composite RGB optical (blue) + WISE $12 \mu \mathrm{m}$ (green) and $22 \mu \mathrm{m}$ (red) image centered on $\kappa$ Ori. We indicate on the right side the XMM-Newton fields observed in Pillitteri et al. (2016) and Pillitteri et al. (2013) with white contours, and the field around V1818 Ori is indicate on the left side. The ring of warm dust is visible in the WISE bands and extends $\sim 2$ deg from $\kappa$ Ori (large dashed circle).

Pillitteri, ObsId 0800190101). The nominal pointing was at $\mathrm{RA}=05^{\mathrm{h}} 53^{\mathrm{m}} 30^{\mathrm{s}}$, Dec $=-10^{\mathrm{d}} 22^{\mathrm{m}} 30.8^{\mathrm{s}}(\mathrm{J} 2000)$ about 3 arcmin offset from the optical position of V1818 Ori. We used EPIC as a prime instrument and the Medium filter. Figure 2 shows a composite RGB image of the XMM-Newton observation in three bands: $0.3-1.0 \mathrm{keV}$ (red), $1.0-3.0 \mathrm{keV}$ (green), and 3.0-8.0 keV (blue).

We used SAS ver. 15 to reduce the data and obtain tables of events calibrated in astrometry, energy, and timing. We selected the events of MOS $1, \operatorname{MOS} 2$, and $p n$ in the $0.3-8.0 \mathrm{keV}$ band, with FLAG $=0$ and PATTERN $<=12$ as prescribed by the SAS guide.

The background was highly variable during the observation, and in order to maximize the signal-to-noise ratio of faint sources, we used the light curve at high energies $(E>10 \mathrm{keV})$ and a cut in rate to select good time intervals and filter out intervals with high background. At this step, we removed about $15 \mathrm{ks}$, leaving thus $25 \mathrm{ks}$ of low-background exposure. This is the portion of exposure time that we used for the subsequent source detection process. The source detection was made with a wavelet convolution technique implemented in a FORTRAN code that finds local maxima in the wavelet-convolved image at different wavelet scales (Damiani et al. 1997a,b). We used a threshold of $4.6 \sigma$ of local background to identify local maxima as X-ray sources. This value should retain at most one spurious source in the image due to statistical fluctuations of the background. We detected 91 point-like sources; we can also recognize by eye two faint extended sources in the northeast corner of the image that have two radio galaxies as likely counterparts.

We searched for infrared counterparts to the X-ray sources in the AllWISE catalog (Wright et al. 2010; Cutri \& et al. 2014) using a matching radius of $5^{\prime \prime}$, and identified $50 \mathrm{X}$-ray sources

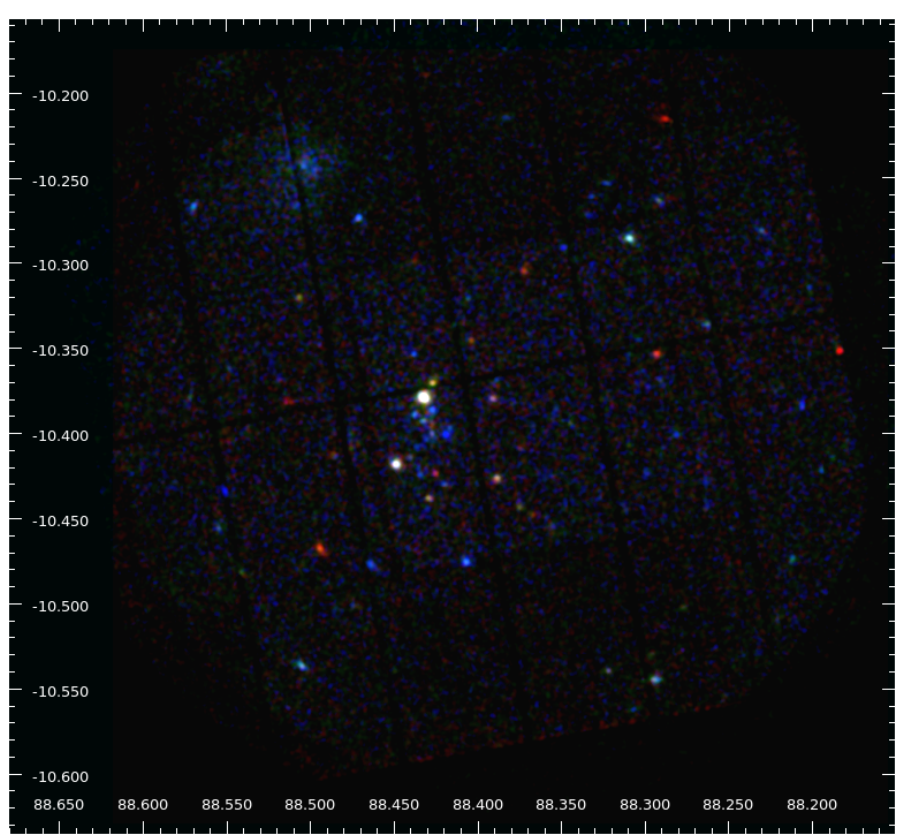

Fig. 2. Composite RGB image of V1818 Ori as observed with XMM-Newton. The color bands are $0.3-1.0 \mathrm{keV}$ (red), $1.0-2.5 \mathrm{keV}$ (green), and 2.5-5.0 keV (blue). About 91 point-like sources and two faint extended sources are detected in the image.

with 50 WISE objects. We supplemented them with the remaining WISE point sources within the XMM-Newton field of view (Fig. 3). The WISE mid-IR color-color and color-magnitude diagrams show sources with distinct IR excesses: $W 2-W 3>$ $1.1 \mathrm{mag}$ and $W 1-W 2>0.3 \mathrm{mag}$. We selected the sources with $W 2 \leq 12$ mag as YSOs; fainter sources are likely to be extragalactic (Koenig et al. 2012; Koenig \& Leisawitz 2014). Two objects with $W 1-W 2>1.5 \mathrm{mag}$ are identified as protostars; the remainder are stars with disks (Class II; Koenig et al. 2012, Fischer et al. 2016). We further identify sources with $W 2-W 3>$ $1.5 \mathrm{mag}, W 2 \leq 12 \mathrm{mag}$, and $W 1-W 2<0.3 \mathrm{mag}$ as transition disks. X-ray sources with $W 2-W 3<1.1$ mag and $W 2 \leq 12$ mag were classified as disk-less pre-main-sequence stars (Class III objects). Another 12 objects with X-ray emission have near-IR colors and magnitudes similar to young premain-sequence stars, that is, no detections in $W 3$ and $W 4$ bands, and $W 1-W 2$ colors fainter than 0.3 mag (Fig. 3); these are classified as Class III objects. Finally, we required $J-K s>$ 0.47 for sources detected in these bands; this eliminated one Class III source with a color bluer than that expected for premain-sequence stars (Fig. 3). The AllWISE images were visually inspected as for the quality flags, signal-to-noise ratio estimates, and $\chi^{2}$ values of the selected YSOs. Two X-ray sources that satisfy the criteria for transition disks are affected by artifacts from V1818 Ori; these are reclassified as Class III objects. Four additional X-ray sources are found with $W 2$ between 13.6 and $14.6 \mathrm{mag}, W 2-W 3<2$ mag (using upper limits in the $W 3$ band), and $J$-band magnitudes fainter than $14.8 \mathrm{mag}$. We excluded them from our catalog for two reasons. First, these stars show $J$-band magnitudes below the hydrogen-burning limit for 3 Myr premain-sequence stars at a distance of up to $400 \mathrm{pc}$ (Baraffe et al. 1998). Second, the spatial positions of these objects are more spread out than the group of YSOs surrounding V1818 Ori, therefore we excluded them as a conservative choice. Future spectroscopic observations are needed to determine whether they truly are pre-main-sequence stars. The remaining X-ray sources 

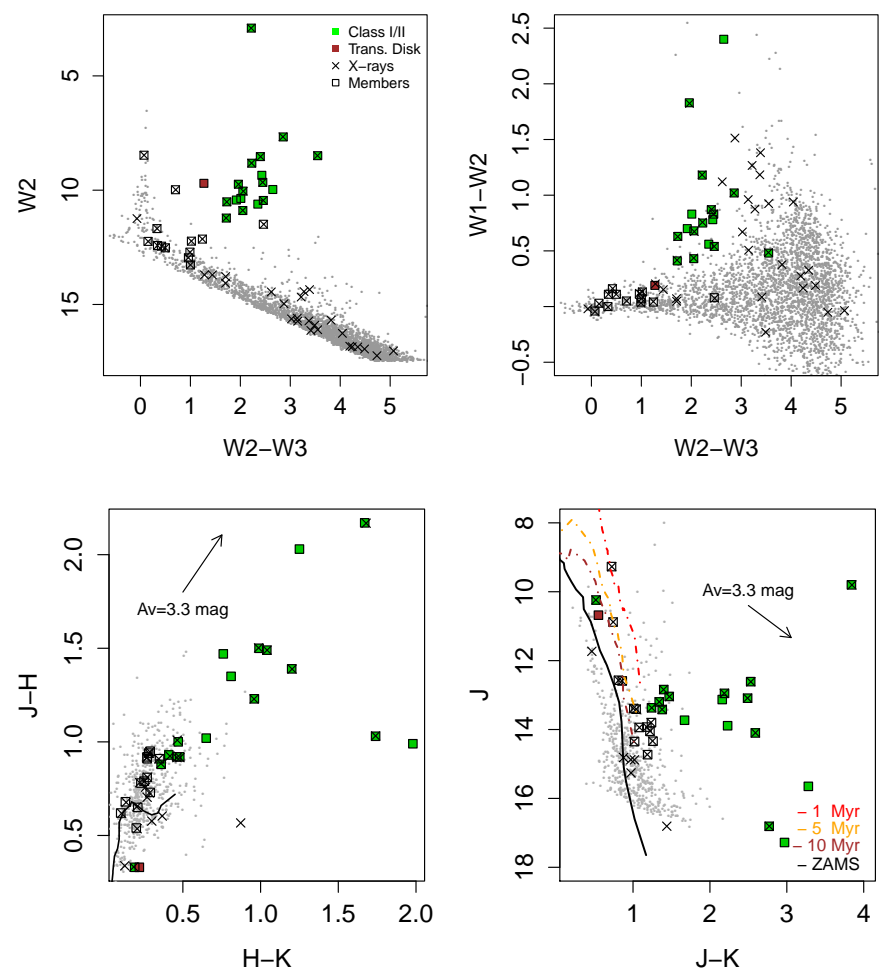

Fig. 3. Top panels: WISE color-color and color-magnitude diagrams of the objects in the XMM-Newton field of view. W1, W2, and W3 refer to the $3.4 \mu \mathrm{m}, 4.6 \mu \mathrm{m}$, and $12 \mu \mathrm{m}$ WISE bands. Selected members of the V1818 Ori group are plotted as open squares. Bottom panels: 2MASS color-color and color-magnitude diagrams. The solid line represents the zero-age main sequence, and dashed lines are isochrones at 1,5 , and $10 \mathrm{Myr}$, respectively, for a distance of $270 \mathrm{pc}$ (the distance moduli for $270 \mathrm{pc}, 400 \mathrm{pc}$, and $900 \mathrm{pc}$ are $\sim 7.2 \mathrm{mag}, \sim 8 \mathrm{mag}$, and $\sim 9.1 \mathrm{mag}$, respectively). The reddening vector corresponding to $A_{\mathrm{V}}=3.3 \mathrm{mag}$ represents the average extinction in the XMM-Newton field.

with IR counterparts are much fainter at $\geq 16$ mag and are not considered to be members. About $41 \mathrm{X}$-ray sources are left without IR counterparts. In Pillitteri et al. (2016) we reported that 47 out of 238 (in two XMM fields) had no IR counterparts, that is, about 23-24 per XMM field. Here we have almost twice the number of X-ray sources without IR matches. An inspection of the XMM image revealed that most of them have blue colors, which means that their spectra are either hard or heavily absorbed. They are scattered in the XMM field of view, while the YSOs are concentrated toward the center. Taken together, the spatial distribution and spectral hardness indicate a background population. The presence of a background cluster of galaxies is not excluded. The two extended sources found in the image could be part of it, although we cannot find a galaxy cluster listed in the 2MASS (Tully 2015) and SDSS catalogs (Tempel et al. 2012) within 20 arcmin from V1818 Ori.

Upper limits to rates were calculated at a threshold consistent with the value used for source detection. The conversion factor (CF) between rates and unabsorbed fluxes $(0.3-8.0 \mathrm{keV}$ band) was derived with PIMMS ver. 4.9. We assumed a thermal coronal spectrum described by an APEC model with $k T=1 \mathrm{keV}$, $Z=0.2 Z_{\odot}$. The average absorption was estimated from an extinction map ${ }^{1}$ (Schlafly \& Finkbeiner 2011), and a value of $E(B-V) \sim 1$, from which we derive $N_{\mathrm{H}}=6.3 \times 10^{21} \mathrm{~cm}^{-2}$

\footnotetext{
1 http://irsa.ipac.caltech.edu/applications/DUST/
}

(corresponding to $A_{\mathrm{V}} \sim 3.3 \mathrm{mag}$ for $R_{\mathrm{V}}=3.1$, Cardelli et al. 1989; the value is reported in the reddening vector in Fig. 3). With these choices, the CF has a value of $2.078 \times 10^{-11} \mathrm{erg} \mathrm{cm}^{-2}$. Locally higher extinction and a different plasma temperature can change the $\mathrm{CF}$ by about $50 \%$. Given the low count statistics of the sources and the short exposure time, it is impossible to perform a more accurate spectral analysis to derive their temperatures and fluxes.

\section{Results and discussion}

We identified 31 members in the V1818 group: 2 protostars (1 with an X-ray detection), 15 Class II sources (11 with $\mathrm{X}$-ray detections), $1 \mathrm{X}$-ray undetected transition-disk object, and 13 X-ray identified Class III sources (Table A.1). Owing to spatial overlap with a nearby source in the ALLWISE data, 1 of the Class II sources without an X-ray detection is considered tentative (see notes to Table A.1). The disk fraction among the YSOs with an X-ray detection is $0.46 \pm 0.14$. Chiang et al. (2015) identified 24 YSOs in the V1818 Ori group using a combination of WISE colors and near-IR observations with the Subaru telescope. Twelve out of 24 YSOs selected by Chiang et al. are in common with our selection of members of the V1818 Ori group. The remaining 12 YSOs are not recovered by our criteria since they have WISE magnitudes below our limits or were identified in deeper near-IR observations with higher angular resolution. Table A.1 lists the 31 YSOs that are identified as members of the V1818 Ori group. These 31 YSOs, including V1818 Ori, form a group of pre-main-sequence stars coincident with the eastern edge of the dust ring surrounding $\kappa$ Ori.

From the fluxes listed in Table A.1, we calculated luminosities, and we used a Kaplan-Meier estimator (as implemented in the R package survival in the case of left-censored data) to assess the distribution of the XLFs of the V1818 Ori group on a set of distances between $115 \mathrm{pc}$ and $1000 \mathrm{pc}$. We used a KolmogorovSmirnov (KS) test to evaluate the significance of the difference between the resulting set of XLFs with the XLF of the YSOs found on the western edge of the $\kappa$ Ori ring. This is a relative distance analysis, but with the assessed distance to the $\kappa$ Ori YSOs in Pillitteri et al. (2016), this analysis will also be calibrated on an absolute scale. The $\kappa$ Ori XLF extends up to $10^{31} \mathrm{erg} \mathrm{s}^{-1}$ and is more skewed than the XLF of V1818 Ori. Since the number of points in the two XLFs differs by a factor of $\sim 8$, we can speculate that the high-luminosity tail in the $\kappa$ Ori XLF might be due to flaring sources or to few foreground objects that contaminate the sample.

The KS statistics peaks at a distance of $\sim 270 \mathrm{pc}$, and the $90 \%$ confidence range is $\sim 230-350 \mathrm{pc}$. For $d=400 \mathrm{pc}$, the KS statistics is $\sim 0.06$, and for $900 \mathrm{pc}$, the KS statistics is $\sim 2 \times 10^{-6}$. A distance of $d=900 \mathrm{pc}$ appears unrealistic, therefore we can safely exclude that V1818 Ori and its surrounding YSOs belong to the Mon R2 association, as has been suggested by Chiang et al. (2015). A distance of $d=400$ pc still appears higher than expected as the two XLFs overlap very marginally at the highluminosity tails, while $d \sim 270$ pc seems the most reasonable. V1818 Ori and the surrounding YSOs seem unrelated to Orion A and L1641 and to the Mon R2 association. They are instead probably part of the $\kappa$ Ori ring because they form a clump inside the eastern edge of it, analogous to the groups of YSOs identified at the western edge of the ring (Pillitteri et al. 2016).

Based on the low background exposure time of $25 \mathrm{ks}$, the completeness limit is estimated above $L_{\mathrm{X}}>10^{29} \mathrm{erg} \mathrm{s}^{-1}$ at a distance of $270 \mathrm{pc}$. This could leave some very low mass stars undetected. Assuming a saturated X-ray luminosity and a ratio 


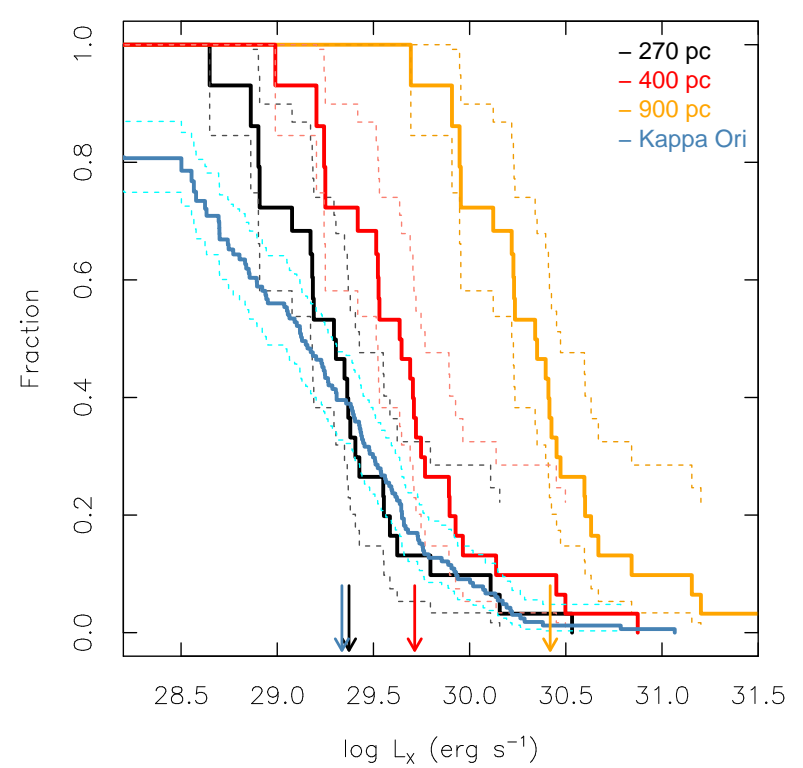

Fig. 4. Kaplan-Meier estimators of the XLF of the YSOs in V1818 Ori calculated for three distances: $900 \mathrm{pc}$ (orange), $400 \mathrm{pc}$ (red), and $270 \mathrm{pc}$ (black). The curves at the $95 \%$ confidence range are plotted as dashed lines. The XLF of the YSOs associated with $\kappa$ Ori (Pillitteri et al. 2016) is shown for comparison. The arrows mark the medians of the different XLFs. The best agreement to the XLF of the $\kappa$ Ori group (distance 250$280 \mathrm{pc}$ ) is obtained for a distance of $270 \mathrm{pc}$.

$L_{\mathrm{X}} / L_{\mathrm{bol}} \sim 10^{-3}$, we have detected stars with $L_{\mathrm{bol}} \geq 10^{32} \mathrm{erg} \mathrm{s}^{-1}$ or about $0.1 L_{\mathrm{bol}, \odot}$. This value corresponds to spectral types of M5, M4, and M2 when adopting isochrones at 1, 5, and $10 \mathrm{Myr}$, respectively (Siess et al. 2000). The fraction of undetected YSOs with IR excesses is about $33 \%$, which alone suggests that the average distance of these objects is unlikely to be $900 \mathrm{pc}$ as for the Mon R2 region. The paucity of protostars and the fraction of stars with disks suggests an age of 2-5 Myr, similar to the distance estimated for the YSOs at the western edge of the ring of $\kappa$ Ori and slightly younger than the age of $\kappa$ Ori itself (Pillitteri et al. 2016).

Considering the whole population of stars within $2 \mathrm{deg}$ of $\kappa$ Ori, its most massive member is $\kappa$ Ori itself (a B0 star), then V1818 Ori is the second massive known member, followed by several low-mass M-type stars. Initial results from an optical spectroscopic survey of the YSOs identified in Pillitteri et al. (2016) reveal that these are predominantly low-mass M-type objects (Pillitteri et al., in prep.). We speculate that the group of YSOs around V1818 Ori is composed mostly of M-type stars as well. Including the sources from Pillitteri et al. (2016), about 152 YSOs formed in the past 2-5 Myr within the $\kappa$ Ori ring,

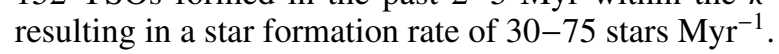

\section{Conclusions}

We have presented the results from the analysis of an XMM-Newton observation of the group of YSOs around the variable young star V1818 Ori, which is associated with the Mon R2 region at 900 pc. However, given that the group is coincident with the eastern edge of the ring of warm dust surrounding $\kappa$ Ori at $\sim 250 \mathrm{pc}$, we investigated their X-ray properties to infer their distances and ages in analogy with the study reported in Pillitteri et al. (2016).

About 91 point-like X-ray sources and two faint extended sources were detected in the $0.3-8.0 \mathrm{keV}$ band. About $50 \mathrm{X}$-ray sources have an infrared counterpart in the WISE catalog. We used AllWISE and 2MASS photometry to identify 31 objects as members of the group, including $25 \mathrm{X}$-ray sources, and classified them as protostars, Class II, Class III, and transition disks. We calculated the X-ray fluxes and luminosities $(0.3-8.0 \mathrm{keV}$ band) for the 25/31 YSOs detected in X-rays and upper limits to fluxes and luminosities for the remaining 6/31. By using a set of different distances to calculate the luminosities and by comparing the resulting XLF to the XLF of the YSOs west of $\kappa$ Ori, we conclude that $900 \mathrm{pc}$ is unrealistic and the group of V1818 Ori is not related to Mon R2. A distance of $400 \mathrm{pc}$ is still marginally consistent with the XLF of the $\kappa$ Ori group, and thus the group does not appear to be related to Orion A and L1641. For a distance of $270 \mathrm{pc}$ (90\% confidence interval in 230-350 pc), we obtain our best agreement with the XLF of the YSOs west of $\kappa$ Ori and the median X-ray luminosity of COUP YSOs. We conclude that V1818 Ori and its surrounding young stars were born in the eastern edge of the $\kappa$ Ori ring and are part of it.

Acknowledgements. I.P. acknowledges support from INAF, ASI and the ARIEL consortium. S.J.W. was supported by NASA contract NAS8-03060 (Chandra $\mathrm{X}$-ray Center). This publication makes use of data products from the Wide-field Infrared Survey Explorer (WISE) and the Two Micron All Sky Survey (2MASS) retrieved through the NASA/IPAC Infrared Science Archive.

\section{References}

Bally, J. 2008, in Handbook of Star Forming Regions, Vol. I, ed. B. Reipurth (ASP Publications), 459

Baraffe, I., Chabrier, G., Allard, F., \& Hauschildt, P. H. 1998, A\&A, 337, 403

Cardelli, J. A., Clayton, G. C., \& Mathis, J. S. 1989, ApJ, 345, 245

Chiang, H.-F., Reipurth, B., \& Hillenbrand, L. 2015, AJ, 149, 108

Connelley, M. S., \& Greene, T. P. 2010, AJ, 140, 1214

Cutri, R. M., et al. 2014, VizieR Online Data Catalog, II/328

Damiani, F., Maggio, A., Micela, G., \& Sciortino, S. 1997a, ApJ, 483, 350

Damiani, F., Maggio, A., Micela, G., \& Sciortino, S. 1997b, ApJ, 483, 370

Fischer, W. J., Padgett, D. L., Stapelfeldt, K. L., \& Sewiło, M. 2016, ApJ, 827, 96

Koenig, X. P., \& Leisawitz, D. T. 2014, ApJ, 791, 131

Koenig, X. P., Leisawitz, D. T., Benford, D. J., et al. 2012, ApJ, 744, 130

Kounkel, M., Hartmann, L., Loinard, L., et al. 2017, ApJ, 834, 142

Megier, A., Strobel, A., Galazutdinov, G. A., \& Krełowski, J. 2009, A\&A, 507, 833

Menten, K. M., Reid, M. J., Forbrich, J., \& Brunthaler, A. 2007, A\&A, 474, 515 Pillitteri, I., Wolk, S. J., Megeath, S. T., et al. 2013, ApJ, 768, 99

Pillitteri, I., Wolk, S. J., \& Megeath, S. T. 2016, ApJ, 820, L28

Schlafly, E., \& Finkbeiner, D. P. 2011, in AAS Meeting Abstracts \#217, BAAS, $43,434.42$

Siess, L., Dufour, E., \& Forestini, M. 2000, A\&A, 358, 593

Tempel, E., Tago, E., \& Liivamägi, L. J. 2012, A\&A, 540, A106

Tully, R. B. 2015, AJ, 149, 171

van Leeuwen, F. 2007, A\&A, 474, 653

Wright, E. L., Eisenhardt, P. R. M., Mainzer, A. K., et al. 2010, AJ, 140, 1868 
I. Pillitteri et al.: XMM-Newton imaging of V1818 Orionis: a young stellar group on the eastern edge of the $\kappa$ Ori ring

\section{Appendix A: Additional material}

Table A.1. Identified YSOs and their 2MASS, WISE, and X-ray photometry.

\begin{tabular}{|c|c|c|c|c|c|c|c|c|c|c|c|c|c|c|c|c|c|}
\hline N. & $\begin{array}{rr}\text { RA } \\
\mathrm{J} 2000 \text { (deg) }\end{array}$ & $\begin{array}{rr}\text { Dec } \\
\mathrm{J} 2000 \text { (deg) }\end{array}$ & designation & $\overline{J J}$ & 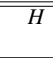 & $\overline{K \mathrm{~s}}$ & $\begin{array}{l}\begin{array}{l}W I \\
\text { mag }\end{array} \\
\end{array}$ & $\overline{W 2}$ & $\overline{~ W 3}$ & $\overline{~ W 4}$ & Classification & Chiang Id & $\begin{array}{l}\text { Rate } \\
\text { ct k }\end{array}$ & $\begin{array}{l}\text { Error } \\
-1\end{array}$ & $\begin{array}{r}\text { Signif } \\
\sigma_{\text {bkg }}\end{array}$ & log Flux & Note \\
\hline 1 & 88.29435 & -10.5443 & J055310.64-103239.6 & 13.9 & 13.0 & 12.8 & 12.5 & 12.4 & 12.1 & 8.08 & Class III & - & 7.09 & 0.55 & 20.60 & -12.83 & \\
\hline 2 & 88.32193 & -10.5394 & J055317.26-103221.8 & 13.4 & 12.5 & 12.1 & 11.6 & 11.2 & 9.5 & 7.67 & Class II & - & 1.27 & 0.24 & 9.10 & -13.58 & \\
\hline 3 & 88.29679 & -10.5301 & J055311.22-103148.2 & 13.8 & 12.8 & 12.6 & 12.4 & 12.2 & 11.2 & 7.89 & Class III & - & 1.41 & 0.30 & 5.90 & -13.53 & \\
\hline 4 & 88.54125 & -10.4813 & J055409.89-102852.7 & 14.1 & 13.1 & 12.8 & 12.6 & 12.4 & 12.0 & 8.72 & Class III & - & 2.12 & 0.46 & 8.80 & -13.36 & \\
\hline 5 & 88.49402 & -10.4679 & J055358.56-102804.3 & 12.6 & 11.9 & 11.8 & 11.7 & 11.7 & 11.3 & 8.64 & Class III & - & 3.45 & 0.36 & 14.30 & -13.14 & \\
\hline 6 & 88.37462 & -10.4431 & J055329.90-102635.1 & 14.7 & 13.8 & 13.5 & 13.3 & 13.3 & 12.3 & 8.93 & Class III & - & 0.85 & 0.14 & 8.10 & -13.75 & \\
\hline 7 & 88.42907 & -10.4383 & J055342.97-102617.9 & 13.0 & 12.0 & 11.6 & 10.7 & 10.0 & 7.99 & 5.84 & Class II & 7 & 1.23 & 0.16 & 12.70 & -13.59 & \\
\hline 8 & 88.38821 & -10.4269 & J055333.17-102536.8 & 13.4 & 12.6 & 12.4 & 12.2 & 12.1 & 10.9 & 7.82 & Class III & - & 1.96 & 0.19 & 17.00 & -13.39 & \\
\hline 9 & 88.42543 & -10.4237 & J055342.10-102525.3 & 12.6 & 11.9 & 11.7 & 11.6 & 11.5 & 9.03 & 7.59 & Class III & - & 0.66 & 0.12 & 7.90 & -13.86 & W3 Contaminated \\
\hline 10 & 88.44868 & -10.4184 & J055347.68-102506.1 & 12.8 & 11.9 & 11.4 & 11.0 & 10.4 & 7.99 & 5.62 & Class II & 21 & 7.91 & 0.40 & 41.80 & -12.78 & \\
\hline 11 & 88.44001 & -10.4141 & J055345.60-102450.7 & 12.9 & 11.7 & 10.8 & 9.4 & 8.53 & 6.13 & 3.62 & Class II & 20 & 0.44 & 0.11 & 5.50 & -14.04 & \\
\hline 12 & 88.48618 & -10.4128 & J055356.68-102446.1 & 13.4 & 12.6 & 12.4 & 12.3 & 12.2 & 12.1 & 8.99 & Class III & - & 0.82 & 0.17 & 6.80 & -13.77 & \\
\hline 13 & 88.37511 & -10.4092 & J055330.02-102432.9 & 10.2 & 9.91 & 9.72 & 8.97 & 8.49 & 4.94 & 2.84 & Class II & 2 & 0.40 & 0.10 & 4.70 & -14.08 & IRAS05510-1025 \\
\hline 14 & 88.42729 & -10.4002 & J055342.54-102400.5 & 9.8 & 7.63 & 5.96 & 4.09 & 2.91 & 0.69 & -1.07 & Class II & 1 & 0.45 & 0.10 & 7.40 & -14.03 & V1818 Ori \\
\hline 15 & 88.41904 & -10.4009 & J055340.57-102403.0 & 12.6 & 11.1 & 10.1 & 8.69 & 7.67 & 4.81 & 1.52 & Class II & 15 & 1.47 & 0.20 & 10.50 & -13.51 & \\
\hline 16 & 88.41640 & -10.3975 & J055339.93-102350.9 & 13.1 & 11.6 & 10.6 & 9.57 & 8.82 & 6.59 & 3.22 & Class II & 14 & 0.25 & 0.08 & 5.00 & -14.29 & \\
\hline 17 & 88.43755 & -10.3894 & J055345.01-102321.9 & 14.1 & 12.7 & 11.5 & 10.5 & 9.66 & 7.21 & 4.39 & Class II & 19 & 0.85 & 0.14 & 9.60 & -13.76 & \\
\hline 18 & 88.39081 & -10.3798 & J055333.79-102247.3 & 13.4 & 12.5 & 12.0 & 11.1 & 10.5 & 8.78 & 7.01 & Class II & 9 & 1.32 & 0.16 & 13.50 & -13.56 & \\
\hline 19 & 88.43200 & -10.3793 & J055343.68-102245.3 & 10.9 & 10.3 & 10.1 & 10 & 9.98 & 9.28 & 6.76 & Class III & - & 18.80 & 0.57 & 72.60 & -12.41 & W3 Contaminated \\
\hline 20 & 88.42676 & -10.3708 & J055342.42-102214.7 & 13.2 & 12.3 & 11.9 & 11.3 & 10.9 & 8.84 & 6.79 & Class II & - & 2.32 & 0.27 & 19.20 & -13.32 & \\
\hline 21 & 88.40408 & -10.3455 & J055336.97-102043.7 & 13.9 & 13.1 & 12.9 & 12.8 & 12.7 & 11.7 & 9.05 & Class III & - & 1.09 & 0.18 & 8.60 & -13.65 & \\
\hline 22 & 88.50644 & -10.3202 & J055401.54-101912.7 & 14.3 & 13.4 & 13.1 & 12.6 & 12.5 & 12.0 & 8.53 & Class III & - & 1.98 & 0.28 & 11.20 & -13.39 & \\
\hline 23 & 88.37238 & -10.3046 & J055329.37-101816.4 & 9.27 & 8.65 & 8.55 & 8.43 & 8.47 & 8.4 & 8.41 & Class III & - & 1.29 & 0.19 & 9.20 & -13.57 & \\
\hline 24 & 88.34882 & -10.2910 & J055323.71-101727.4 & 16.8 & 15.8 & 14.0 & 11.6 & 9.74 & 7.78 & 5.57 & Protostar & - & 0.84 & 0.16 & 7.70 & -13.76 & \\
\hline 25 & 88.43061 & -10.1900 & J055343.34-101124.0 & 14.3 & 13.6 & 13.3 & 13.1 & 13 & 12.0 & 8.34 & Class III & - & 1.11 & 0.30 & 4.60 & -13.64 & \\
\hline 26 & 88.34597 & -10.2923 & J055323.03-101732.2 & 17.3 & 16.3 & 14.3 & 12.4 & 9.97 & 7.32 & 3.68 & Protostar & - & $<0.84$ & - & - & $<-13.76$ & \\
\hline 27 & 88.38743 & -10.4534 & J055332.98-102712.2 & 15.7 & 13.6 & 12.4 & 11.2 & 10.4 & 8.35 & 5.59 & Class II & 7 & $<0.45$ & - & - & $<-14.03$ & \\
\hline 28 & 88.29652 & -10.5440 & J055311.16-103238.3 & 10.7 & 10.3 & 10.1 & 9.89 & 9.7 & 8.43 & 6.6 & Trans. Disk & - & $<7.09$ & - & - & $<-12.83$ & \\
\hline 29 & 88.35579 & -10.4543 & J055325.39-102715.6 & 13.9 & 12.4 & 11.7 & 11.1 & 10.4 & 8.51 & 6.12 & Class II & 5 & $<0.46$ & - & - & $<-14.02$ & Tentative \\
\hline 30 & 88.44192 & -10.5811 & J055346.06-103452.1 & 13.7 & 12.7 & 12.1 & 11.2 & 10.6 & 8.26 & 6.19 & Class II & - & $<1.04$ & - & - & $<-13.67$ & \\
\hline 31 & 88.35442 & -10.4584 & J055325.06-102730.1 & 13.1 & 11.8 & 11 & 10.1 & 9.35 & 6.92 & 4.8 & Class II & 4 & $<0.48$ & - & - & $<-14.00$ & \\
\hline
\end{tabular}

Notes. Objects in common with Chiang et al. (2015) are indicated. The detection significance is given in units of the local background $\sigma$. Unabsorbed fluxes are given in the $0.3-8.0 \mathrm{keV}$ band. For undetected YSOs, the rates and fluxes are upper limits. Notes indicate the contamination from spikes or bright nearby objects and the names of objects reported in the literature. 